FredericQ, P. (1958). J. gen. Microbiol. 18, 527-528

\title{
Colicins, Golicinogenic Factors and their Relations to Bacteriophages
}

\author{
By P. FREDERICQ \\ University of Liège
}

Many enteric bacteria, mostly Escherichia coli and Shigella sonnei, but also other Shigella spp. and some Salmonella spp., produce a variety of diffusible antibiotic substances, named colicins. All colicins appear to be of a protein nature but differ in many other properties: extent and specificity of their activity spectra, specificity of resistant mutants, diffusibility, morphology of the inhibition zones in agar, susceptibility to proteolytic enzymes, thermoresistance, electrophoretic motility, antigenic properties. Their activity spectra are strikingly different but strictly limited to other strains of the Enterobacteriaceae family. Susceptibility-patterns are controlled by a number of receptors specific of each colicin to which a strain is susceptible. Susceptible bacteria are rapidly killed by the colicin which they adsorb.

The presence of the colicin-receptors is governed by genetic factors which may recombine by crossing or get lost by mutation. These mutations are spontaneous and independent of the other properties. They are specific of each receptor, since a mutant resistant to a given colicin is still inhibited by other colicins acting on the mother-strain. It is, however, possible to transform a strain susceptible to many colicins into a completely resistant one if all its specific receptors are removed successively in a series of mutations.

Colicins display unexpected relations with bacteriophages. Colicinogenic strains of bacteria resemble lysogenic strains, and colicins behave, in many respects, like bacteriophages. Activity spectra of both agents are quite comparable in their diversity, and susceptibility-patterns are controlled in each case by a number of specific receptors, some of which may be common to a phage and a given colicin. The bactericidal activity of colicins is comparable to that of virulent bacteriophages, more particularly to their killing action without multiplication of the particles. In fact, the bactericidal components of some virulent bacteriophages must be substances related to colicins. For example, the bactericidal component of coliphage $\mathbf{T} 6$ is a protein which has the same specificity as colicin $\mathbf{K}$, adsorbs on the same receptor and displays the same sensitivity to $\mathrm{X}$-ray inactivation.

Colicins and bacteriophages are, however, agents of a fundamentally different nature. Colicins are chemical substances, proteins, which kill bacteria without being reproduced, whereas bacteriophages are biological units, endowed with genetic continuity, which are reproduced and multiplied in the cells they kill. Bacteriophages are much more complex particles which include a deoxyribonucleic core and a protein envelope.

The genetic structure which is included in the phage particles and induce their 
reproduction is absent from colicins. Owing to the hereditary stability and specificity of the colicinogenic properties, such genetic structures must, however, be present in the bacteria which produce the colicins. These genetic factors can be demonstrated by the fact that they may be transmitted from one strain to another. As they are not set free by lysis of the cells, their transfer requires the mechanism of sexual conjugation, but they behave, in recombination experiments, as if completely independent of the normal genetic structure of the bacteria.

Like prophages in lysogenic strains, colicinogenic factors are potential lethal agents whose spontaneous or induced development kills the cells in which it occurs. They are also pathogenic, although their pathogenicity is only disclosed by the achievement of their potentiality. As long as they remain in a latent condition, they induce, on the other hand, immunity to the corresponding colicin. Colicinogenic factors are transmissible pathogenic agents which are independent of the normal genetic structure of bacteria and might therefore be regarded as bacterial viruses, distinct from bacteriophages. Their relations with the latter may perhaps point to a common parental ancestry. 